\title{
Perspective liquid scintillators for spectrometry of neutron and gamma radiation
}

\author{
Kawa Haji Mahmoud ${ }^{1}$, Vaclav Prenosil ${ }^{1}$, Zdenek Matej ${ }^{1}$, Frantisek Kucera ${ }^{2}$, Filip Mravec ${ }^{3}$, Michal \\ Kostal $^{4}$, Frantisek Cvachovec ${ }^{5}$, David Valis ${ }^{5}$
}

\begin{abstract}
This paper presents some results of the development of two new scintillation detectors usable for the detection and spectrometry of neutron and photon components of the mixed field. It is a series of liquid organic scintillators containing carbon, hydrogen, nitrogen and oxygen. Elements of individual scintillator designs are arranged in various chemical bonds defining physical and chemical properties of the scintillators. These scintillators have advantageous useful properties. They are non-hygroscopic, not poisonous and are not sensitive to contact with the outside atmosphere and can be stored in conventional laboratory containers.
\end{abstract}

Keywords - scintillators, scintillation detectors, pulse shape discrimination, Figure of merit

\section{INTRODUCTION}

The authors list basic properties of two types of new liquid scintillators LSB205 and LSB206 that are compared with the characteristics of the BC $501 \mathrm{~A}$ (NE-213) liquid scintillator, which can be considered the standard used for many years for detection and spectrometry in the mixed field of neutron and photon radiation. Characteristic data of the scintillators developed are focused on the energy range useful, e.g. reactor dosimetry, i.e., neutron energy $\leq 15 \mathrm{MeV}$. This paper mainly contains qualitative and quantitative data on the resolution of neutron and photon particles detected by the scintillator. The resolution of the charged particles is done by pulse shape discrimination, which is based on a variant of the integration method [1], [2]. Quantitatively, the quality of the resolution of charged particles is characterized by the generalized function of Figure of Merit - FOM [2], [3]. If FOM $>1$, neutron and gamma radiation responses are perfectly separated. If FOM $<1$ then the responses of both particles overlap. Besides, the article contains data on energy resolution and light output of scintillator. The interesting data were observed when investigating the relations of characteristics in a temperature range of $0-50{ }^{\circ} \mathrm{C}$. In order to use these scintillators for gamma/neutron mixed field spectrometry, it is necessary to obtain light output

This work was supported in part by the Ministry of Industry and Trade, within the project TRIO, No. FV20453.

1 Masaryk University, Botanická 15, Brno 612 00, Czech Republic

2 Faculty of Chemistry, Brno University of Technology, Purkyňova 464/118, 612 function at least for protons. The obtained scintillator temperature dependencies described in this article will enable to refine the measurement of the amplitude resolution of the neutron spectrum measured at the collimated reactor output beam moderated by silicon. This spectrum contains several neutron lines with well-defined energies which could substitute hard to obtain mono-energetic neutron sources [4], [5]. The next step towards the usability for neutron and photon spectrometry in the mixed field is the calculation of the response functions needed for the unfolding of energy spectra.

The properties of newly developed liquid scintillators give them the potential to be used in various areas of nuclear physics, including nuclear safety.

\section{DESCRIPTION OF THE MEASURING OBJECT}

There are many types of scintillators for mixed neutron and gamma radiation fields. The main requirement for such a scintillator is the ability to distinguish between neutron and gamma radiation interactions. In this paper, we want to focus on comparing the temperature dependencies of the standard BC-501A scintillator and two versions of the newly developed LSB 205 to LSB 206 scintillators containing 88.3 to $91.1 \% \mathrm{C} ; 7.6$ to $8.7 \%$ $\mathrm{H} ; 0.1$ to $1.9 \% \mathrm{~N}$ and 0.1 to $2.2 \% \mathrm{O}$ in the $\mathrm{CF}^{252}$ source pool.

\section{METHODOLOGY}

We compare the thermal properties of the experimental scintillators and the standard liquid scintillator BC-501A. This scintillator has very good separation properties. However, it loses its properties when exposed to air oxygen. The composition of the liquid scintillators tested is not public - it is still protected.

\section{EXPERIMENTAL SETUP}

The NGA-01 spectrometric system was used for the measurement. This two-parameter system uses

\footnotetext{
3 VF, a.s. Svitavská 588, 67921 Černá Hora, Czech Republic 4 Research Center Rez Ltd, 25068 Husinec-Rez 130, Czech Republic

5 University of Defence, Kounicova 65, Brno 612 00, Czech Republic
} 
2" PMT Hamamatsu R329-02 equipped with an active tapered voltage divider as a detector. This configuration allows the device to be used even with large amplitude ranges of input signals while maintaining excellent output linearity. This configuration ensures excellent results even at high count rates per second. The device can measure up to 700,000 pulses per second.

The NGA-01 measures detector output pulses using two differently amplified branches for two 12bit ADC inputs with a sample rate of $500 \mathrm{MS} / \mathrm{s}$ - see Figure 1. Two channels are used for better signal-tonoise ratio, especially for smaller amplitudes. The data are digitally filtered, summed and evaluated by Field Programmable Gate Array (FPGA).

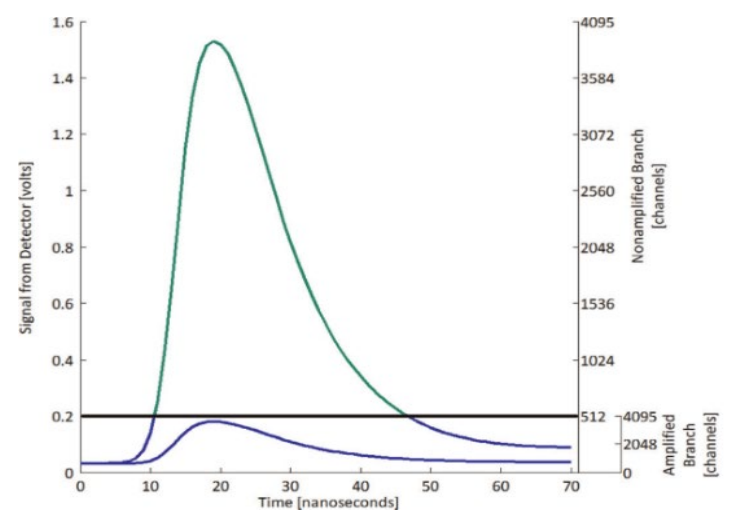

Fig. 1. Amplified and non-amplified impulse selection [3], [6]

The $\mathrm{Cs}^{137}$ and $\mathrm{Co}^{60}$ emitters were used for calibration of the measurement. The Compton edges in the photon instrument spectrum are for the $\mathrm{Cs}^{137}$ a $0.477 \mathrm{MeV}$ energy edge and for the $\mathrm{Co}^{60}$ emitter a $1.17 \mathrm{MeV}$ energy edge.

For all scintillators, we used the same PSD (pulse shape discrimination) method based on Charge Comparison [1], [2] implemented directly in the FPGA of the spectrometer NGA-01.

The individual pulse parameters used in the relationship to quantify the PSD are 'energy delta' and 'discrimination delta' values are determined empirically for optimal delimitation of areas with maximum neutron and photon responses in the scintillator being measured.

\section{DESCRIPTION OF THE TEMPERATURE CHAMBER}

The Vötsch Industrietechnik GmbH VT4004 type chamber was used for temperature dependence testing. Temperatures of $0,5,10,20,30,40$ and 50 degrees Celsius were used for the experiments. A photomultiplier with an active resistor divider was placed in the chamber. The measuring apparatus was outside the air-conditioned space. The connection was made by coaxial cables. The interior of the temperature chamber was shielded from ambient light.

All tested scintillators were air-conditioned at the same time. Calibration sources and test source were mounted on scintillators from their side.

\section{TEMPERATURE TEST RESULTS}

The results of the experiments were CSV matrices expressing temperature dependence of individual scintillators. The FOM temperature dependencies of each scintillator for the selected test temperature repertoire and concrete energy are shown in the following graphs for each scintillator separately.

We chose a range of 0.5 to $4.5 \mathrm{MeV}$ gamma to display temperature dependencies of separation quality. This area of energy is interesting for our further research.

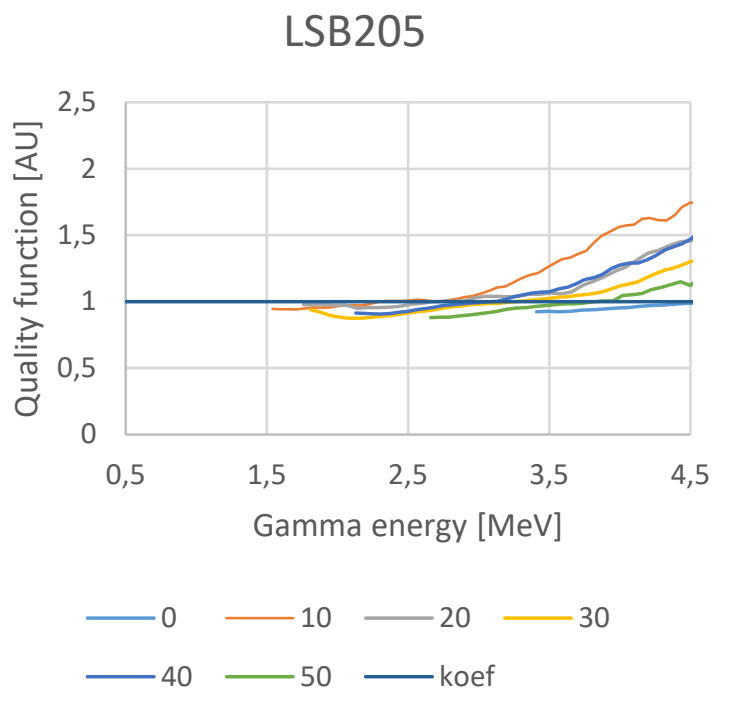

Fig. 2. FOM temperature dependencies of the LSB 205 scintillator

The LSB205 scintillator is not fully satisfactory parameters. It does not register all particles below $1.5 \mathrm{MeV}$, and therefore it is not possible to evaluate the FOM. It provides reliable separation of particle responses in the order over $3 \mathrm{MeV}$.

The influence of temperature on the FOM is substantial. It best separates at temperatures around 10 degrees Celsius. For other temperatures, the quality of separation is considerably worse.

The LSB206 scintillator has better parameters. It reliably registers particles with energies below 0.5 $\mathrm{MeV}$, and thus the FOM can be evaluated in the range of selected energies. It provides reliable separation of particle responses in the order of up to $2 \mathrm{MeV}$.

The influence of temperature on the FOM is again substantial. It best separates at temperatures around 10 degrees Celsius. For other temperatures, the 
quality of separation is considerably worse.

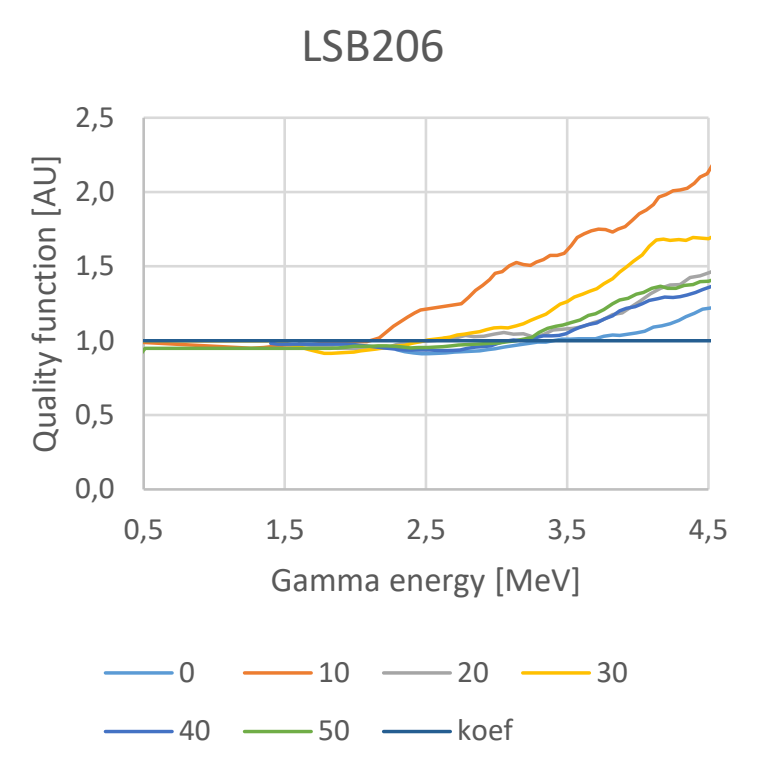

Fig. 3. FOM temperature dependencies of the LSB 206 scintillator

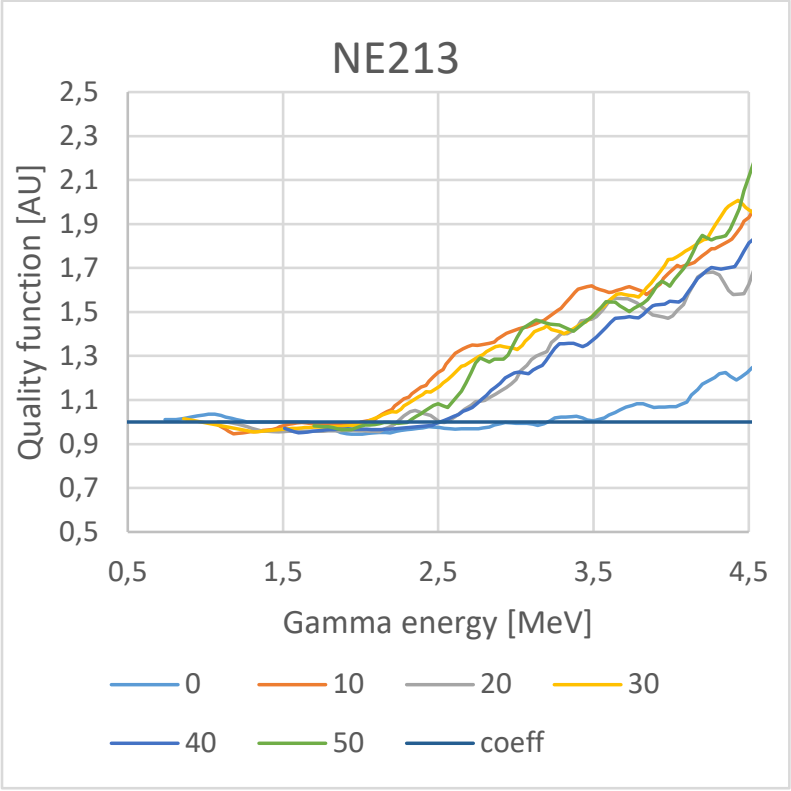

Fig. 4. FOM temperature dependencies of the NE213 scintillator

The NE213 scintillator is basically the liquid scintillator standard for neutron and gamma radiation separation. The dependence of the FOM on the temperature is shown in FIG. 4. Has the best parameters from the studied scintillators. It reliably registers particles with energies below $0.5 \mathrm{MeV}$, and thus the FOM can be evaluated in the range of selected energies. It provides reliable separation of particle responses in the order of up to $2 \mathrm{MeV}$.

The influence of temperature on the FOM is again substantial. NE213 scintillator worst separates at temperatures around 0 degrees Celsius. For other temperatures, the quality of separation is essentially the same.

Differences in scintillator behavior will be the subject of further research and experimentation.

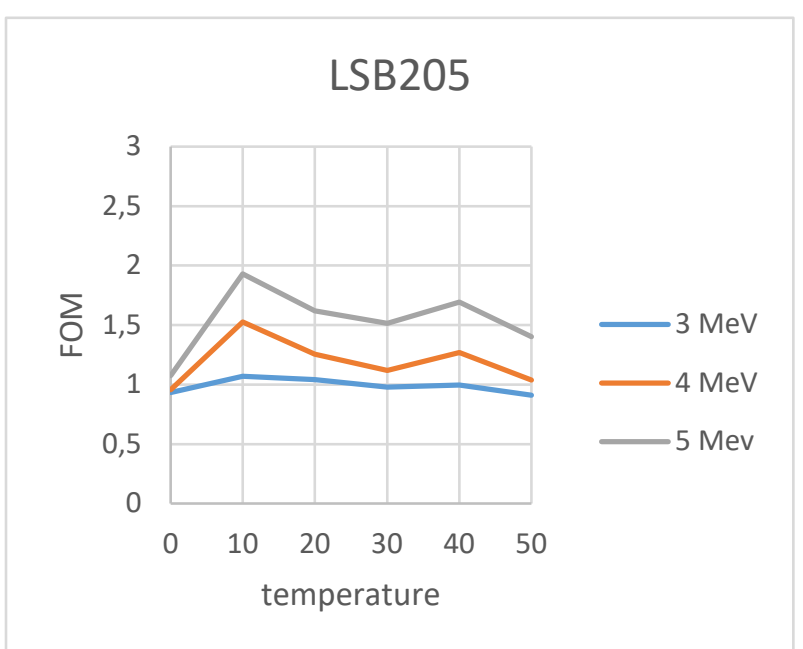

Fig. 5. FOM temperature dependence of the LSB 205 for some energy

The graphs of Figures 5 to 7 show the temperature dependence on the generalized function of Figure of Merit for three particle energies. Specifically for 3, 4 and $5 \mathrm{MeV}$ gamma. It is clear from the graphs that the effect of temperature on FOM has a similar course for all scintillators studied.

The effect of temperature on the FOM of the scintillators LSB205 and LSB206 is most pronounced for 10 degrees Celsius and then decreases. More markedly decreases for the scintillator LSB206.

The temperature effect on the NE213 FOM scintillator is most pronounced for 0 degrees Celsius and then almost constant.

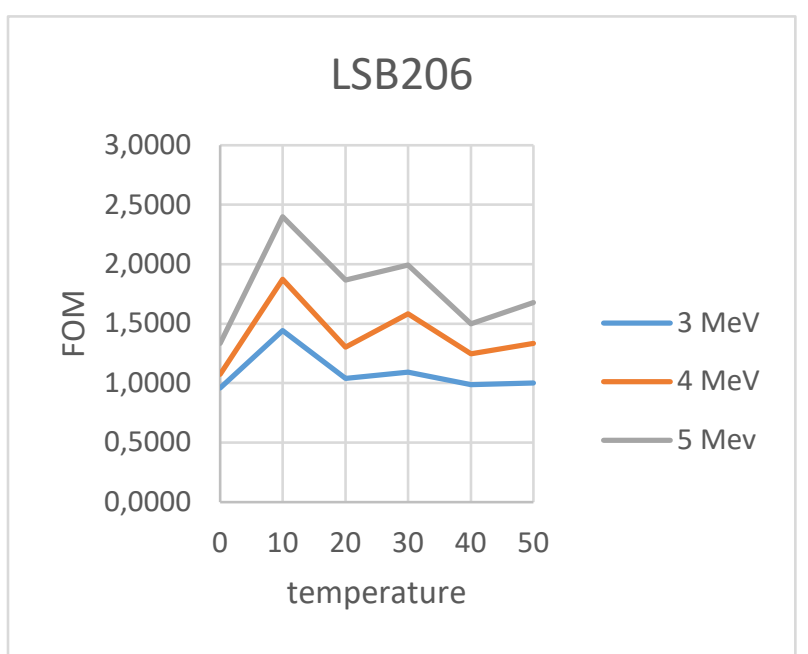

Fig. 6. FOM temperature dependence of the LSB 206 for some energy 


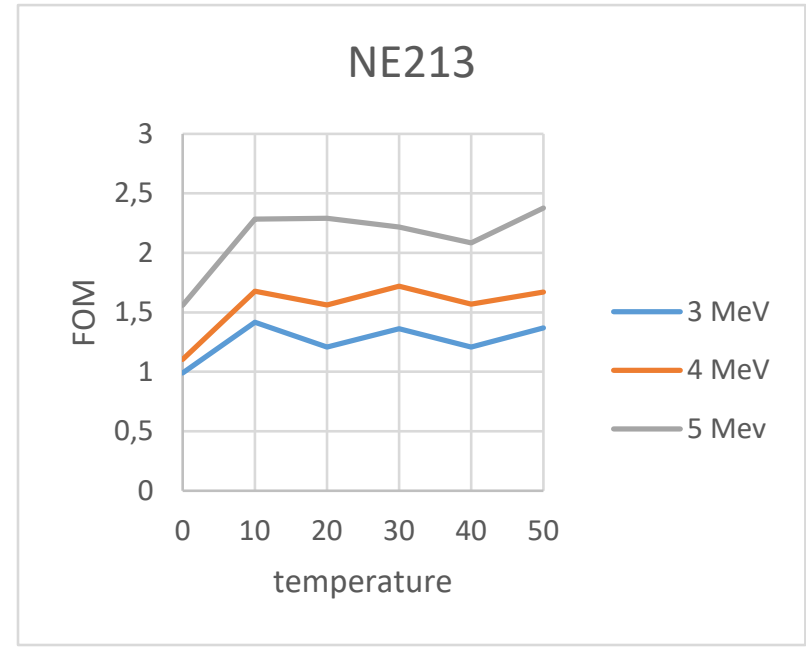

Fig. 7. FOM temperature dependence of the NE213 for some energy

\section{CONCLUSION}

It can be seen from the graphs that the FOM temperature dependence is neither linear nor monotonic. The reason for the non-linearity will be the subject of further research. Knowledge of the FOM temperature dependence of scintillators allows eliminating this dependence in calculations of energy spectra of measured neutron-gamma mixed radiation.

\section{REFERENCES}

[1] M. Amiri, V. Prenosil, F. Cvachovec, Z. Matej, and F. Mravec. "Quick algorithms for real-time discrimination of neutrons and gamma rays," Journal of Radioanalytical and Nuclear Chemistry, 2015; 303(1): 583-599. Published online 2014 Aug 24. Doi: 10.1007/s10967-014-3406-5

[2] Z. Matej, M. Amiri, F. Cvachovec, V. Prenosil, F. Mravec, Z. Kopecky, "Digital two-parametric processing of the output data from radiation detectors," Progress in Nuclear Science and Technology. Volume 4 (2014) pp. 670-674. DOI: $10.15669 /$ pnst.4.670

[3] M. Amiri, F. Cvachovec, Z. Matej, V. Prenosil, F. Mravec, "Digital data processing of stilbene," 3rd International Conference on Advancements in Nuclear Instrumentation, Measurement Methods and their Applications (ANIMMA). June 2013. DOI: 10.1109/ANIMMA.2013.6728010

[4] M. Kostal, M. Schulc, J. Soltes, E. Losa, L. Viererbl, Z. Matej, F. Cvachovec, V. Rypar, "Measurements of neutron transport of well-defined silicon filtered beam in lead. "Applied Radiation and Isotopes, Elsevier, 2018, roč. 2018, č. 142, s. 160-166.

[5] M. Kostal, Z. Matej, E. Losa, O. Huml, M. Stefanik, F. Cvachovec, M. Sulc, B. Jansky, E. Novak, D. Harutyunyan, V. Rypar, "On similarity of various reactor spectra and 235U prompt fission neutron spectrum,“ Applied Radiation and Isotopes, Elsevier, 2018, roč. 135, May, s. 83-91.

ISSN 0969-8043 doi: 10.1016/j.apradiso.2018.01.028

[6] M. Pavelek, Z. Matej, O. Herman, F. Mravec, M. Veskrna, F. Cvachovec, M. Kostal, V. Prenosil, "Fast Digital Spectrometer for Mixed Radiation Fields,“. 2017 IEEE SENSORS Proceedings. IEEE, 2017. s. 436-438, 3 pp. ISBN 978-1-5386-4056-2.

doi:10.1109/ICSENS.2017.8234012 